\title{
Femtosecond pulse generation with voltage-controlled graphene saturable absorber
}

\author{
I. Baylam, ${ }^{1,2}$ M. N. Cizmeciyan, ${ }^{1,2}$ S. Ozharar, ${ }^{3}$ E. O. Polat, ${ }^{4}$ C. Kocabas, ${ }^{4}$ and A. Sennaroglu ${ }^{1,2, *}$ \\ ${ }^{1}$ Laser Research Laboratory, Departments of Physics and Electrical-Electronics Engineering, Koç University, \\ Rumelifeneri, Sarıer, Istanbul 34450, Turkey \\ ${ }^{2}$ Koç University Surface Science and Technology Center (KUYTAM), Rumelifeneri, Sarıyer, Istanbul 34450, Turkey \\ ${ }^{3}$ College of Arts and Sciences, Bahçeşehir University, Beşiktaş, Istanbul 34353, Turkey \\ ${ }^{4}$ Bilkent University, Department of Physics, Ankara 06800, Turkey \\ ${ }^{*}$ Corresponding author: asennar@ku.edu.tr
}

Received June 9, 2014; accepted June 20, 2014;

posted July 30, 2014 (Doc. ID 213698); published August 27, 2014

\begin{abstract}
We report, for the first time to our knowledge, the demonstration of a graphene supercapacitor as a voltagecontrolled saturable absorber for femtosecond pulse generation from a solid-state laser. By applying only a few volts of bias, the Fermi level of the device could be shifted to vary the insertion loss, while maintaining a sufficient level of saturable absorption to initiate mode-locked operation. The graphene supercapacitor was operated at bias voltages of $0.5-1 \mathrm{~V}$ to generate sub-100 fs pulses at a pulse repetition rate of $4.51 \mathrm{MHz}$ from a multipass-cavity $\mathrm{Cr}^{4+}$ :forsterite laser operating at $1255 \mathrm{~nm}$. The nonlinear optical response of the graphene supercapacitor was further investigated by using pump-probe spectroscopy. (c) 2014 Optical Society of America

OCIS codes: (140.4050) Mode-locked lasers; (140.5680) Rare earth and transition metal solid-state lasers; (140.3460) Lasers; (140.7090) Ultrafast lasers; (160.4236) Nanomaterials.

http://dx.doi.org/10.1364/OL.39.005180
\end{abstract}

The two-dimensional graphene crystal, with its nearly constant absorption over a broad wavelength range, possesses many favorable optical and electronic properties [1] and has been successfully used as a saturable absorber to generate femtosecond pulses from numerous lasers in the near- and mid-infrared [2-4]. In modelocking applications, one drawback stems from the fact that even for monolayer graphene, the small signal roundtrip insertion loss is of the order of 5\%, which makes it quite challenging to operate low-gain lasers (such as Cr:forsterite and colquiriites) with graphene saturable absorbers. One possible remedy involves using supercapacitor structures containing graphene so that, when a voltage bias is applied, the Fermi level can be shifted to reduce the passive losses, while maintaining a sufficient level of saturable absorption to initiate pulse generation.

Graphene-based solid-state capacitors with voltagedependent variable absorption have been previously demonstrated as saturable absorbers, but their operation was limited to infrared wavelengths $[\underline{5}, \underline{6}]$. This is primarily because bias levels necessary to vary the amount of visible absorption were high enough to cause dielectric breakdown in the capacitor structure. More recently, it has been shown that graphene-based supercapacitors are more advantageous, since, owing to the use of an electrolyte, the Fermi level can be more readily shifted to vary the absorption up to the visible region, even with low bias voltages of the order of several volts [7]. An additional advantage of the supercapacitor structure is that no complex microfabrication stage is required in the preparation of the device in contrast with the dielectric-based graphene capacitor structures.

In this Letter, we report the first demonstration of a graphene supercapacitor employed as a voltagecontrolled graphene saturable absorber (VCG-SA) for the generation of sub-100 fs pulses. The graphene supercapacitor was fabricated as a modulator and introduced into a $\mathrm{Cr}^{4+}$ :forsterite laser operated at $1255 \mathrm{~nm}$. The bias of the VCG-SA was varied in the $0-3 \mathrm{~V}$ range to determine the optimum operating conditions of the mode-locked laser. Mode-locked operation could be initiated for bias voltages in the range of $0.5-1 \mathrm{~V}$. At the bias voltage of $1 \mathrm{~V}$, 84 fs pulses with a time-bandwidth product of 0.32 and peak power of $37 \mathrm{~kW}$ were generated near the center wavelength of $1255 \mathrm{~nm}$. We also investigated the dependence of the saturation fluence, modulation depth, and linear loss of the VCG-SA on the applied voltage.

In the experiments, the graphene supercapacitor was fabricated by using two monolayer large-area graphene electrodes, each synthesized via chemical vapor deposition and transfer printed onto a $1 \mathrm{~mm}$ thick, $1 \mathrm{in}$. diameter quartz substrate. The $100 \mu \mathrm{m}$ spacing between the two graphene electrodes was then filled with an electrolyte (Diethymethyl (2-methoxyethyl) ammonium bis (trifluromethylsulfony) imide [DEME][TFSI]), which has a relative dielectric constant of $\epsilon_{r}=14$ [8]. A sketch of the graphene super capacitor is shown in Fig. 1(a). The electrical connections to the graphene electrodes were made by using carbon tapes. Under no bias voltage, the Fermi level $E_{F}$ resides at the Dirac point, as seen in Fig. 1(b), and interband excitation of the electrons leads to broadband absorption of photons, which is approximately $1.5 \%$ per graphene electrode. The application of a voltage bias forms electrical double layers (EDLs) in the vicinity of positive and negative graphene electrodes. Due to the electrostatic doping of electrons, the Fermi level of the negative graphene electrode shifts up, as shown in Fig. $1(\mathrm{c})$. In this case, the amount $E_{F}$ of the shift is given by

$$
E_{F}=\hbar \nu_{F} \sqrt{\pi n},
$$

where $\nu_{F}$ is the Fermi velocity $\left(1.1 \times 10^{8} \mathrm{~cm} / \mathrm{s}\right), n$ is the electron density, and $\hbar$ is the Planck's constant [7]. 


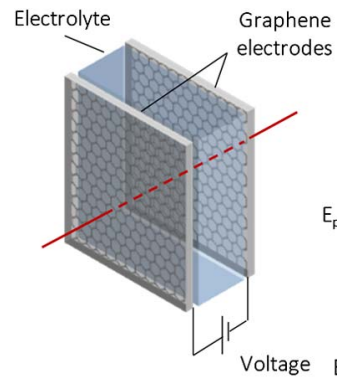

(a)
$V=0$

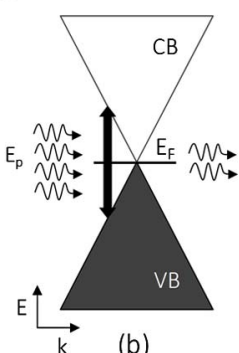

$\vee \neq 0$

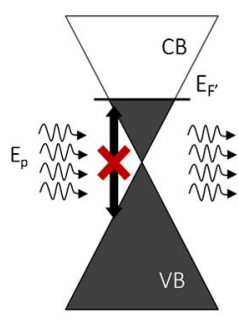

(c)
Fig. 1. (a) Schematic of the VCG-SA based on the supercapacitor structure. Schematic representation of the band structure of (b) unbiased $(V=0)$ and (c) biased $(V \neq 0)$ graphene, where $V$ is the applied bias voltage, $E_{P}$ is the photon energy, $E_{F}$ is the Fermi energy, and $\mathrm{CB}$ and $\mathrm{VB}$ are the conduction and valance bands, respectively.

Due to Pauli blocking, this shift in the Fermi level completely suppresses the absorption of the device for wavelengths $(\lambda)$ with photon energy below $2\left|E_{F}\right|$; in other words, for $\lambda<h c / 2\left|E_{F}\right|$. The resulting voltage-dependent change in the optical transmission occurs at relatively low bias voltages of a few volts due to the use of an electrolyte with a high dielectric constant.

The voltage-dependent change in the optical transmission of the VCG-SA is further delineated in Fig. 2(a). In this figure, $\Delta T / T_{0}$ is the normalized change in the transmission with respect to the transmission $T_{0}$ at zero bias.

From Fig. 2(a), the maximum change in the fractional transmission of two graphene layers was determined to be $3 \%$ at $1200 \mathrm{~nm}$. Figure 2(b) further shows the variation
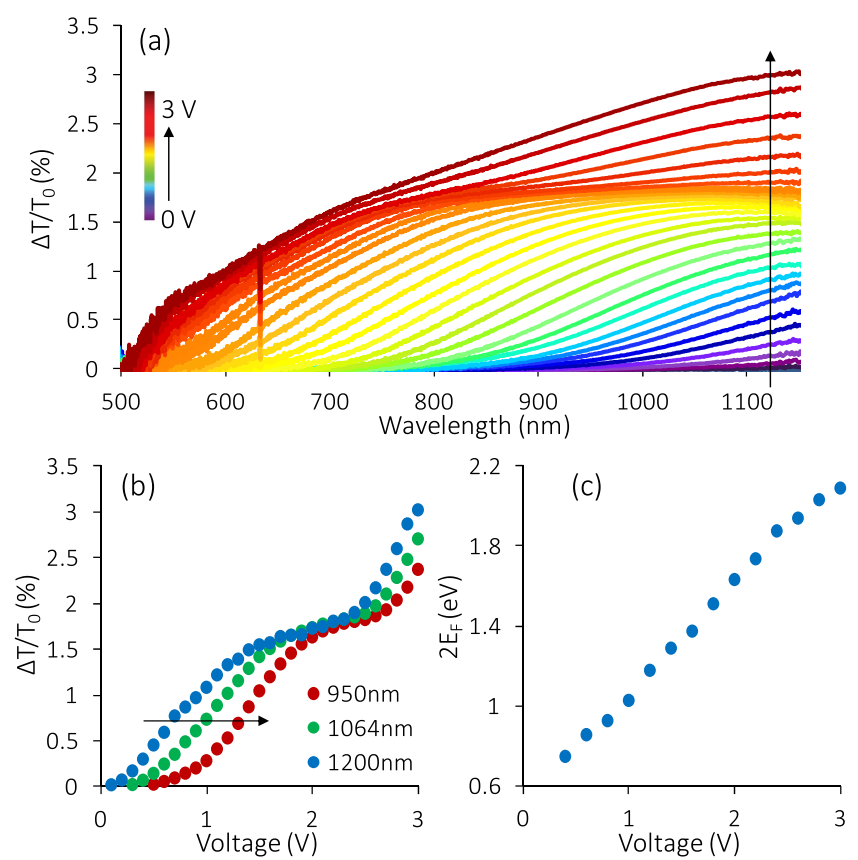

Fig. 2. (a) Variation of the normalized change of the optical transmission of the graphene as a function of the wavelength at different bias voltages in the $0-3 \mathrm{~V}$ range. (b) Measured variation of the fractional change in the optical transmission as a function of the bias voltage at the wavelengths of 950, 1064, and $1200 \mathrm{~nm}$. (c) Estimated Fermi level shift of the device as a function of the bias voltage. of the normalized transmission at 950, 1064, and $1200 \mathrm{~nm}$ as a function of the bias voltage. In the actual supercapacitor, a more complicated two-step response of the transmission was observed as a function of the applied bias voltage, which probably could be attributed to the asymmetric ionic strengths and different ionic radii of the cations and anions of the electrolyte [7, $]$ ]

Figure 2(c) shows the variation of the effective Fermi energy shift of the device as a function of the bias voltage, which was deduced from the fractional optical transmission data. The effective Fermi energy shift linearly scales with the applied voltage, meaning that higher applied bias voltages are required to modulate the transmission at shorter wavelengths. With this specific electrolyte, the effective Fermi level of the device could be shifted by up to $1.2 \mathrm{eV}$ at a voltage bias of only $3 \mathrm{~V}$, enabling the use of this supercapacitor as a modulator down to the wavelength of $500 \mathrm{~nm}$ in the visible range.

The ultrafast and nonlinear optical responses of the VCG-SA were investigated by using a time-resolved pump probe spectrometer (HELIOS, Ultrafast Systems). As a pump source, we used the output of a tunable optical parametric amplifier (TOPAS, Spectra Physics) at $700 \mathrm{~nm}$, which was pumped with $2 \mathrm{~mJ}, 100 \mathrm{fs}$ pulses at a pulse repetition rate of $1 \mathrm{kHz}$ from a commercial Ti:sapphire chirped-pulse amplifier (Spitfire ACE, Newport-Spectra Physics).

The sample was probed at $1250 \mathrm{~nm}$. Pump-probe measurements depicted in Fig. 3(a) show that, up to an applied voltage of $1 \mathrm{~V}$, the typical ultrafast response of the graphene can be seen with average fast and slow time constants of 283 fs and 1.9 ps. For voltages beyond $1 \mathrm{~V}$, however, the device had more complicated dynamics, possibly because of the mixed role of intraband and interband transitions at $1250 \mathrm{~nm}$ [1]. Figure $\underline{3(\mathrm{~b})}$ also shows that the device acts a saturable absorber as the fluence of light is increased. Our measurements,
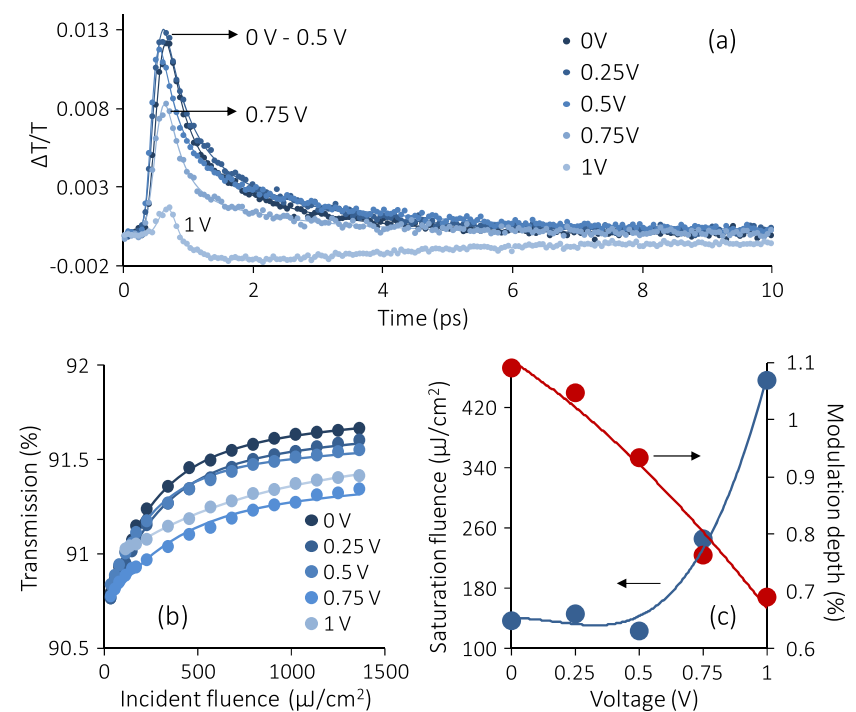

Fig. 3. (a) Ultrafast response of the graphene-based supercapacitor at the probe wavelength of $1250 \mathrm{~nm}$ for different bias voltages. (b) Measured change of the optical transmission as a function of the incident light fluence at $700 \mathrm{~nm}$. (c) Measured variation of the saturation fluence and modulation depth at $1250 \mathrm{~nm}$ as a function of the bias voltage. 
summarized in Fig. 3(c), further showed that the saturation fluence at $1250 \mathrm{~nm}$ monotonically increased with applied bias, becoming $456 \mu \mathrm{J} / \mathrm{cm}^{2}$ at $1 \mathrm{~V}$. Furthermore, the modulation depth was found to decrease from $1.09 \%$ at $0 \mathrm{~V}$ to $0.69 \%$ at $1 \mathrm{~V}$ at $1250 \mathrm{~nm}$. In the mode-locking experiments, it was not possible to use this device as an effective saturable absorber near $1255 \mathrm{~nm}$ for bias voltages above $1 \mathrm{~V}$.

For mode-locking experiments, we used a roomtemperature, multipass-cavity (MPC) $1250 \mathrm{~nm} \mathrm{Cr}^{4+}$ :forsterite laser, the schematic of which is shown in Fig. 4. The oscillator, which is similar to the setup described in [11], was end pumped with a Yb fiber laser at $1064 \mathrm{~nm}$, and the output coupler had a transmission of $2.4 \%$ at $1250 \mathrm{~nm}$. The cavity was further extended with an MPC to reduce the repetition rate to $4.51 \mathrm{MHz}$. Such a resonator architecture enables the scaling of the pulse energy at low average output powers and eliminates undesirable thermal effects in the laser gain medium [12]. The VCG-SA was placed between two curved high reflectors with radius of curvature of $50 \mathrm{~cm}$ to obtain sufficient fluence on the device to initiate mode-locked operation.

The estimated beam waist on the graphene sample was $97 \mu \mathrm{m}$. The device was further positioned at a Brewster angle to minimize reflection losses and to prevent FabryPerot etalon effects during mode-locked operation.

Figure $\underline{5}$ shows the continuous-wave power performance of the laser oscillator without VCG-SA and with VCG-SA operating at different voltages. The slope efficiency without VCG-SA was around $6.7 \%$, giving a maximum output power of $350 \mathrm{~mW}$ at $8 \mathrm{~W}$ of input power. After the insertion of the VCG-SA into the cavity, the slope efficiency decreased to $0.5 \%$ at zero bias. However, as the applied voltage was increased, the shift in the Fermi level reduced the passive losses, and the slope efficiency increased up to $1.6 \%$ at $3 \mathrm{~V}$.

Figure 6(a) also shows the variation of output power of the laser as a function of the applied bias voltage at the pump power of $7.1 \mathrm{~W}$. As the bias voltage was increased, the output power increased from 11 to $69 \mathrm{~mW}$. By using the fact that the threshold pump power is proportional to the sum of passive losses and the output coupler transmission of the resonator, we estimated the voltage dependent loss of the VCG-SA as depicted in Fig. 6(b)

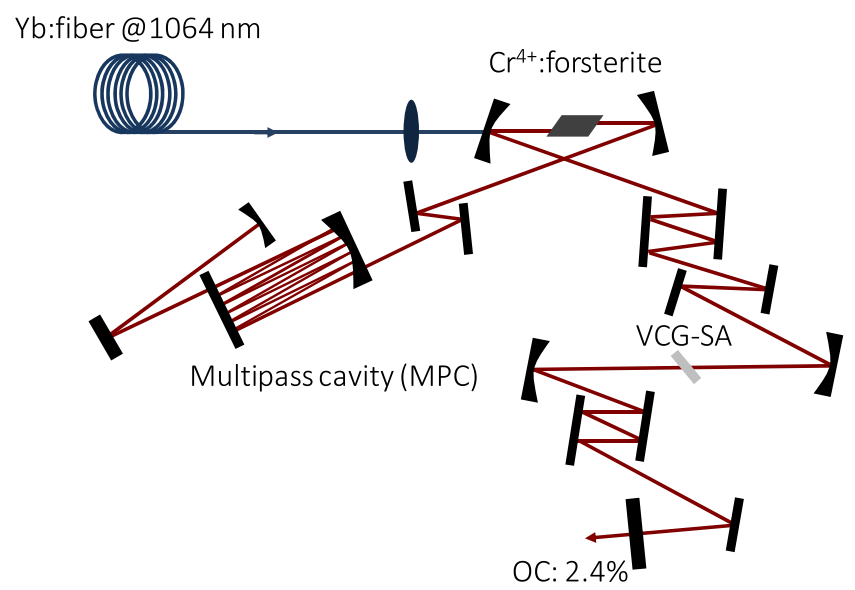

Fig. 4. Experimental setup of the multipass-cavity $\mathrm{Cr}^{4+}$ : forsterite laser mode-locked with the VCG-SA.

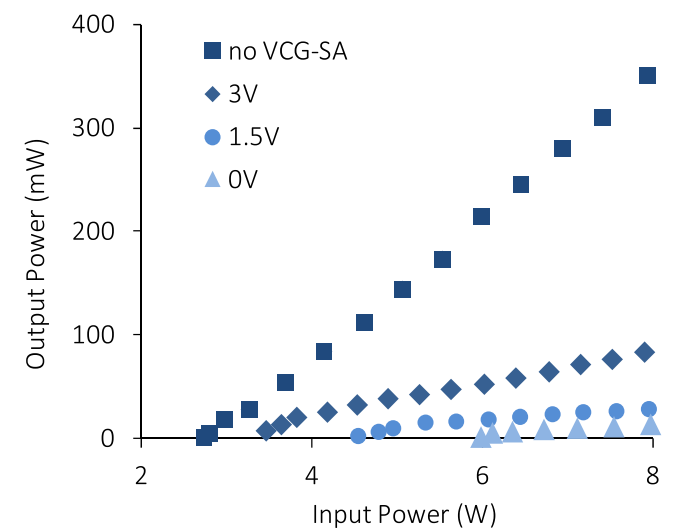

Fig. 5. Efficiency curve of the laser with and without the VCG sample.
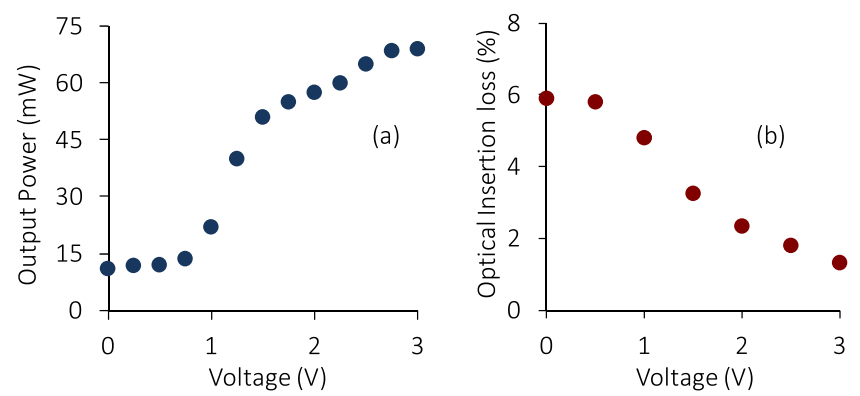

Fig. 6. (a) Measured change of the output power of the $\mathrm{Cr}^{4+}$ : forsterite laser as a function of the applied voltage at the pump power of $7.1 \mathrm{~W}$. (b) Estimated single pass optical insertion loss of the VCG-SA at different bias voltages.

[11]. As can be seen, the single-pass optical insertion loss of the modulator decreased from $5.9 \%$ at zero bias to $1.3 \%$ at $3 \mathrm{~V}$ of bias.

For bias voltages between 0.5 and $1 \mathrm{~V}$, single-pulse mode-locked operation of the laser could be successfully obtained by translating the output coupler. Below this range, the high insertion loss of the VCG-SA resulted in insufficient intracavity power; hence, no mode-locked operation could be observed. Although the laser could generate femtosecond pulses for applied voltages above $1 \mathrm{~V}$, the fixed resonator dispersion was not sufficient to balance the nonlinearities, and the output spectrum contained a continuous-wave spike.

Figures 7 and 8 summarize the mode-locking results, including the autocorrelation traces, the mode-locked spectra, and the RF spectra of the generated pulses at the bias voltages of 0.5 and $1 \mathrm{~V}$. The resonator produced $89 \mathrm{fs}$ (84 fs) pulses with a time-bandwidth product of 0.35 $(0.32)$ and a peak power of $30 \mathrm{~kW}(37 \mathrm{~kW})$ at the applied bias of $0.5 \mathrm{~V}(1 \mathrm{~V})$. In both cases, the pulses were nearly transform-limited, and the sideband noise of the photodetected pulse train was determined to be $63 \mathrm{dBc}$ $(70 \mathrm{dBc})$ below the carrier at a resolution bandwidth of $1 \mathrm{kHz}$. These results further show that the VCG-SA device simply operates as a fast saturable absorber switch to initiate mode locking in each case ( 0.5 or $1 \mathrm{~V})$, and the eventual mode-locking performance of the laser, which is nearly the same in both cases, only depends on the cavity dispersion and nonlinearity. 

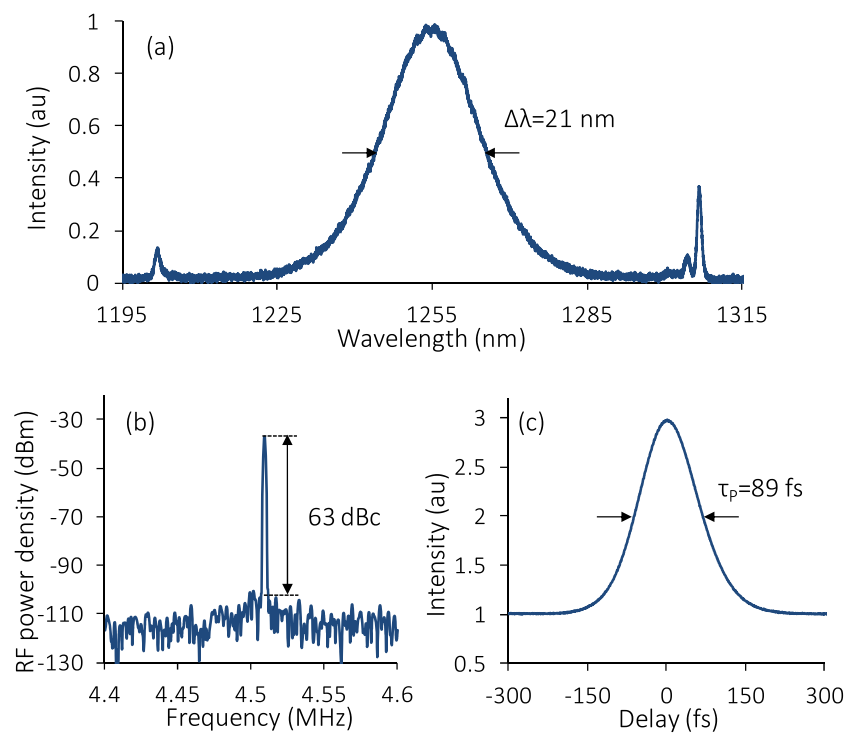

Fig. 7. (a)-(c) Spectrum, autocorrelation trace, and RF spectrum ( $1 \mathrm{kHz}$ resolution bandwidth) of the generated pulses at $1256 \mathrm{~nm}$ at the bias voltage of $0.5 \mathrm{~V}$.
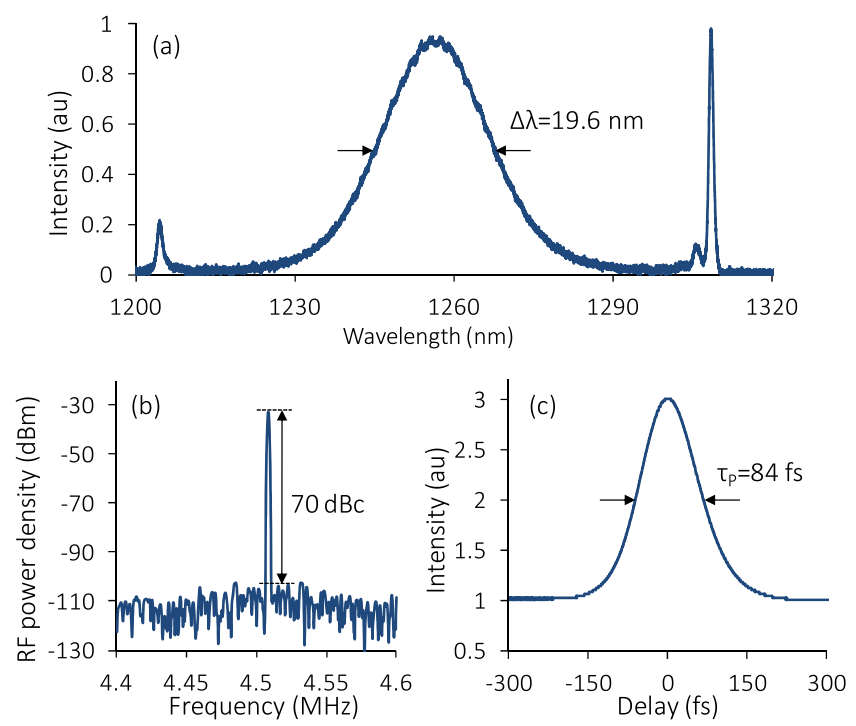

Fig. 8. (a)-(c) Spectrum, autocorrelation trace, and RF spectrum ( $1 \mathrm{kHz}$ resolution bandwidth) of the generated pulses at $1255 \mathrm{~nm}$ at the bias voltage of $1 \mathrm{~V}$. The pulse repetition frequency of the mode-locked oscillator was $4.51 \mathrm{MHz}$.

At the highest single-pulse output power, the maximum intracavity fluence on the sample was estimated to be $364 \mu \mathrm{J} / \mathrm{cm}^{2}$ at $0.5 \mathrm{~V}\left(424 \mu \mathrm{J} / \mathrm{cm}^{2}\right.$ at $\left.1 \mathrm{~V}\right)$. For these intracavity fluence levels, no damage was observed on the sample.

In conclusion, we report the first demonstration of femtosecond pulse generation by using a graphene-based supercapacitor saturable absorber. One of the most favorable characteristics of the graphene supercapacitor, in comparison with its solid-state counterparts, is the possibility of shifting the Fermi level by several eV (in our case $\left|2 E_{F}\right|$ by as much as $2.4 \mathrm{eV}$ ) with relatively low bias voltages of the order of several volts. In our experiments conducted with a multipass cavity $\mathrm{Cr}^{4+}$ :forsterite laser, we could successfully generate sub-100 fs, nearly transform-limited pulses near $1255 \mathrm{~nm}$. In comparison with other conventional nanostructured carbon-based saturable absorbers employed in previous studies $[11,13]$, the most important advantage of the current scheme is that the proposed graphene supercapacitor architecture enables the flexibility of adjusting the overall device loss with reasonably low bias voltages while still enabling mode-locked operation. We believe that this superior characteristic makes the VCG-SA device a potentially important candidate for modelocking a wide range of low-gain lasers in the visible and near-infrared.

\section{References}

1. F. Bonaccorso, Z. Sun, T. Hasan, and A. C. Ferrari, Nat. Photonics 4, 611 (2010).

2. H. Baek, H. W. Lee, S. Bae, B. H. Hong, Y. H. Ahn, D.-I. Yeom, and F. Rotermund, Appl. Phys. Express 5, 032701 (2012).

3. Z. Sun, T. Hasan, and A. C. Ferrari, Physica E 44, 1082 (2012).

4. M. N. Cizmeciyan, J. W. Kim, S. Bae, B. H. Hong, F. Rotermund, and A. Sennaroglu, Opt. Lett. 38, 341 (2013).

5. C. C. Lee, S. Suzuki, W. Xie, and T. R. Schibli, Opt. Express 20, 5264 (2012).

6. C. C. Lee, C. Mohr, J. Bethge, S. Suzuki, M. E. Fermann, I. Hartl, and T. R. Schibli, Opt. Lett. 37, 3084 (2012).

7. E. O. Polat and C. Kocabas, Nano Lett. 13, 5851 (2013).

8. M.-M. Huang, Y. Jiang, P. Sasisanker, G. W. Driver, and H. Weingärtner, J. Chem. Eng. Data 56, 1494 (2011).

9. R. M. Lynden-Bell, A. I. Frolov, and M. V. Fedorov, Phys. Chem. Chem. Phys. 14, 2693 (2012).

10. L. M. Malard, K. F. Mak, A. H. C. Neto, N. M. R. Peres, and T. F. Heinz, New J. Phys. 15, 015009 (2013).

11. S. Ozharar, I. Baylam, M. N. Cizmeciyan, O. Balci, E. Pince, C. Kocabas, and A. Sennaroglu, J. Opt. Soc. Am. B 30, 1270 (2013).

12. A. Sennaroglu, A. M. Kowalevicz, E. P. Ippen, and J. G. Fujimoto, IEEE J. Quantum Electron. 40, 519 (2004).

13. I. Baylam, S. Ozharar, H. Cankaya, S. Y. Choi, K. Kim, F. Rotermund, U. Griebner, V. Petrov, and A. Sennaroglu, Opt. Lett. 37, 3555 (2012). 